\title{
Wavelet Neural Modeling for Hydrologic Time Series Forecasting with Uncertainty Evaluation
}

\author{
Yan-Fang Sang • Zhonggen Wang • Changming Liu
}

Received: 4 July 2014 / Accepted: 28 December 2014 /

Published online: 16 January 2015

(C) Springer Science+Business Media Dordrecht 2015

\begin{abstract}
An approach, with the basic idea of resampling wavelet neural parameters, was proposed for probabilistic forecasting of hydrologic time series by the wavelet neural model. Parameters in wavelet neural model are assumed as following uniform distribution, and both proper convergence criterion and likelihood function are used to train the wavelet neural structure and judge the acceptance of parameter set. By training and learning wavelet neural structure as many times (i.e., resampling neural parameters) until becoming stable, all sets of wavelet neural parameters are composed as the resampling results, based on which probabilistic forecasting of hydrologic time series is attained. Optimal forecasting result can be gained by computing mathematical mean of the resampling results, and uncertainty can be described by proper confidence interval. Results of one runoff example indicated the identical performance of the proposed approach and wavelet regression model, but both perform better than conventional neural model. The proposed approach has similar efficiency as the Bayesian method for uncertainty evaluation, and both show higher efficiency than traditional MonteCarlo method. Choice of proper convergence criterion is an important task when using the proposed approach, because it directly determines the convergence rate, accuracy and uncertainty level of probabilistic forecasting result. Overall, several key issues should be carefully considered for obtaining more reasonable probabilistic forecasting results by the proposed approach, including choice of proper likelihood function, accurate wavelet decomposition of series, and determination of proper wavelet neural structure.
\end{abstract}

Keywords Hydrologic time series analysis · Probabilistic forecasting - Uncertainty evaluation · Wavelet neural model $\cdot$ Monte-Carlo simulation

Y.-F. Sang $(\bowtie) \cdot$ Z. Wang $\cdot$ C. Liu

Key Laboratory of Water Cycle \& Related Land Surface Processes, Institute of Geographic Sciences and Natural Resources Research, Chinese Academy of Sciences, Beijing 100101, China e-mail: sangyf@igsnrr.ac.cn

Yan.-Fang. Sang

e-mail: sunsangyf@gmail.com 


\section{Introduction}

Hydrologic time series forecasting is an important task to understand the future hydrologic regime, and further guide practical water activities (Sivakumar et al. 2001; Tiwari and Chatterjee 2010). Black-box models are used commonly for the task (Jain and Kumar 2007; Nourani et al. 2009). They use certain mathematical tools to describe the correlations of hydrologic variables, but do not require detailed knowledge of physical hydrologic processes (Ki i 2009b). Linear regression models are the typical black-box models and develop extensively (Box and Jenkins 1976; Ki i 2010). However, they cannot describe nonlinear and nonstationary variability of hydrologic processes (Kwon et al. 2006). Artificial neural network (ANN) is another important type of black-box models (Maier and Dandy 2000). ANN model can learn complex nonlinear relationship of hydrologic variables (Luk et al. 2000), also in cases where such relationship is unknown or cannot be made explicit a priori (Alvisi and Franchini 2011). Many studies have proved the better performance of ANNs compared with linear regression models.

Wavelet analysis can elaborate the localized characteristics of a series both in temporal and frequency domains, so it can describe nonstationary variability of hydrologic processes (Torrence and Compo 1998; Sang 2013). Presently, the combination of wavelet analysis with conventional neural models has become a prevalent approach to conduct hydrologic time series forecasting (Coulibaly et al. 2000; Ramana et al. 2013). For wavelet neural model inputs, original series are usually decomposed into a set of sub-signals by discrete wavelet transform (Mallat 1989; Danbechins 1992). Each sub-signal takes a different proportion in original series and has a distinctive behavior. Various studies indicate the better performance of this practice due to its nonlinear and nonstationary adaption (Anctil and Tape 2004; Nourani et al. 2008; Ki i 2009a; Maheswaran and Khosa 2014).

Those wavelet neural models used presently have been almost exclusively deterministic. However, hydrologic process like any other natural processes has uncertainty (Arabi et al. 2007; Tang et al. 2007; Geza et al. 2009). Because many models are subjected to inherent model and parameter uncertainties (Alvisi and Franchini 2011), the hydrologic forecasting result with single optimal value does not take uncertainty into account effectively, and is not convincing (Krzysztofowicz 1999). Hydrologic forecasting is a statement of an uncertain future value of the hydrologic variable of interest (Kumar and Maity 2008; Sang et al. 2010). A model cannot eliminate the uncertainty associated with a future event, but at most reduce it (Krzysztofowicz 2001). Consequently, techniques for handling uncertainty should be considered in the development of wavelet neural models.

Generally, the uncertainty factors influencing hydrologic forecasting include data uncertainty (Bormann 2008; Biemans et al. 2009), model structure uncertainty (Tung and Mays 1981; Krzysztofowicz 1999), and model-parameter uncertainty (Kaheil et al. 2006; Sang et al. 2010; van Werkhoven et al. 2009). When applying a certain wavelet neural model to hydrologic forecasting, it usually assumes that statistical properties of the hydrologic series are temporal persistent. Once parameters are determined, they are assumed to remain constant over time. This is often not a valid assumption, because climate and other factors influencing hydrologic dynamics may cause changes in the statistical properties of series over time (Kumar and Maity 2008). On the other hand, there are usually a large number of parameters with equifinality in wavelet neural model. The concept of equifinality can be understood as: there are various sets of wavelet neural parameters that are consistent with the observed data, but no single one is consistently better than others, so parameter interaction and sensitivity must be handled implicitly (Beven and Freer 2001; Vrugt et al. 2009). Consequently, model-parameter 
is a key factor influencing the reliability and uncertainty of hydrologic time series forecasting by wavelet neural model.

In this study, the main objective is to propose an approach for probabilistic forecasting of hydrologic time series by wavelet neural model. By employing the concept of equifinality (Beven and Binley 1992), we can obtain a "resampling results" (as defined afterward) for wavelet neural forecasting. The optimal forecasting result can be gained by computing mean value of the resampling results, and uncertainty of forecasting result can be evaluated by describing the statistical characters of the resampling results, based on which probabilistic forecasting by wavelet neural model can be done. The rest contents of this paper are organized as follows. Section 2 briefly describes discrete wavelet decomposition of series. Section 3 proposes the approach for probabilistic forecasting of hydrologic time series. Section 4 applies the approach to a runoff example for verification. Finally, a set of understandings and conclusions summarize the study.

\section{Discrete Wavelet Decomposition of Series}

Wavelet analysis methods are widely used for hydrologic time series analysis (Wang et al. 2011). Observed hydrologic series in nature are usually discrete series. They can be analyzed by discrete wavelet transform (DWT) given proper mother wavelet $\psi(t)$ (Percival and Walden 2000):

$$
W_{f}(j, k)=\int_{-\infty}^{+\infty} f(t) \psi_{j, k}{ }^{*}(t) d t \text { with } \psi_{j, k}(t)=a_{0}{ }^{-j} / 2 \psi\left(a_{0}{ }^{-j} t-b_{0} k\right)
$$

where $f(t)$ is the series analyzed, and $t$ is a time index; $a_{0}$ and $b_{0}$ are constants, integer $j$ is decomposition level, and $k$ is time translation factor; $\psi^{*}(t)$ is the complex conjugate. In practice, the dyadic DWT is used commonly by assigning $a_{0}=2$ and $b_{0}=1$ (Danbechins 1992):

$$
W_{f}(j, k)=\int_{-\infty}^{+\infty} f(t) \psi_{j, k}{ }^{*}(t) d t \text { with } \psi_{j, k}(t)=2^{-j / 2} \psi\left(2^{-j} t-k\right)
$$

More details of the mathematical foundation of dyadic DWT were thoroughly described in (Mallat 1989). Sub-signal $f_{j}(t)$ of original series $f(t)$ under level $j$ can be reconstructed as:

$$
f_{j}(t)=\sum_{k} W_{f}(j, k) \psi^{*}\left(2^{-j} t-k\right)
$$

and their sum is original series:

$$
f(t)=\sum_{j=1}^{M} f_{D j}(t)+f_{A M}(t)
$$

where $M$ is the decomposition level used; $f_{D j}(t)$ is the sub-signal under level $j$ reconstructed by detail coefficients, and $f_{A M}(t)$ is the sub-signal under level $M$ reconstructed by approximation coefficients.

Sang (2012) established a reference energy function for discrete wavelet analysis, and proposed a step-by-step guide to discrete wavelet decomposition of series. Specifically, it is to choose appropriate wavelet according to the statistical characters relationship among original 
series, de-noised series and removed noise, choose proper decomposition level by analyzing the difference between energy function of the analyzed series and the reference energy function, and then identify deterministic components by conducting significance testing of DWT. The guide is used in this paper for accurate discrete wavelet decomposition of series, as the basis of wavelet neural modeling.

\section{Uncertainty Evaluation Approach for Wavelet Neural Modeling}

Neural model is supposed to possess the capability to reproduce the unknown nonlinear relationship existing between a set of input explanatory variables and output forecasted variables (Shrestha and Solomatine 2006). Back propagation neural network (BPNN) is one well-known neural model. The key problem about BPNNs is how to accurately determine network structure. The number of input layer nodes is usually determined according to the correlations between explanatory input variables and forecasted variables (Cheng et al. 2008). The number of hidden layer nodes is usually determined by the "trail and error" procedure (i.e., sensitivity analysis) (Kumar and Maity 2008). The number of output layer nodes is determined by the research object.

Compared with conventional artificial neural models, a wavelet neural model is constructed in which accurate wavelet decomposition results of original input series are the input data vector, and original output series are still the output data vector. The approach proposed for probabilistic forecasting of hydrologic time series by wavelet neural model is explained as follows:

(1) Choose proper wavelet and decomposition level, and decompose the hydrologic series by conducting significance testing of DWT. To be specific, for the series $f(t)$ to be analyzed, it is decomposed into a set of sub-signals $\left\{f_{D 1}(t), f_{D 2}(t), \ldots, f_{D M}(t), f_{A M}(t)\right\}$ with the total number of $M+1$ given proper wavelet and decomposition level $M$, as described in Eq. (4);

(2) Choose suitable numbers of input-, mid- and output-layer nodes, and determine the structure of wavelet neural model. The structure of wavelet neural model is constructed in which sub-signals of original input data at $t$ time are as the input of neural model, and original data series at $t+\tau$ time are as the output data of neural model, where $\tau$ is the time delay;

(3) Use the same explanatory input data and output data vectors to train the wavelet model structure. A new set of wavelet neural parameters can be gotten in each training time, and the corresponding simulation result can also be obtained using the new parameter set;

(4) Repeat step (3) many times. When statistical characters (including mean value and standard deviation) of simulation results do not change along with the training time increase, we think that the results become stable, and the training of wavelet model structure is finished. The final results of these sets of wavelet neural parameters are called "resampling results";

(5) Probabilistic forecasting of hydrologic time series is done based on the resampling results. The optimal forecasting result is gained by computing mean value of the resampling results, and uncertainty of forecasting result is evaluated using the confidence interval with proper confidence level (e.g., $95 \%$ ).

Two key problems should be carefully considered when using the approach. One is the choice of proper convergence criterion. These neural parameters of weights and biases in 
wavelet neural model are assumed as following uniform distribution here, and no more conditions are given to constrain their value ranges. However, in the training process of wavelet neural model structure, there is an assigned convergence criterion (e.g., 0.001), which indicates the difference between observed data and modeled data, and determines model's capacity. The convergence criterion can restrain the value range of neural parameter. If lapsing far from optimal parameters values, modeling result would not pass the convergence criterion. Therefore, a proper convergence criterion should be firstly chosen when using the proposed approach. The other problem is the choice of proper likelihood function. Likelihood function is employed to judge the acceptance of each resampling result. Besides the convergence criterion, physical meaning of the forecasted hydrologic variable should also be considered when judging the acceptance of modeling result. When analyzing runoff series in Section 4, Eq. (5) is used as likelihood function to judge each resampling result:

$$
L F=\min \left(y^{t+\tau}\right) \geq 0
$$

in which $y^{t+\tau}$ is the output value. Equation (5) clearly restrains that forecasting runoff data must be not smaller than zero. Only the forecasting results meeting both the convergence criterion and Eq. (5) are acceptable. When analyzing other hydrologic variables, the likelihood function should be determined according to its specifically physical meaning.

\section{Case Study}

\subsection{Data}

A monthly runoff series data (RS1) measured at the Xixian hydrologic station in east China is used as example of wavelet neural modeling. The Xixian station locates at the upstream of the Huai River watershed and controls a drainage area of $6659.96 \mathrm{~km}^{2}$ (Fig. 1). The RS1 series has a duration of 41 years, from the 1961 to 2001 . The first 30 years of data (73\% of the whole data set) are used for calibration, and the remaining 11 years (27\% of the whole data set) are used for verification. All analysis practices about the runoff data are conducted in MathWorks MATLAB R2010.

Statistical characters of the data sets are presented in Table 1. The data sets in calibration and verification periods show similar scattered variation $\left(C_{v}=1.43\right.$ and 1.54$)$, positive skewness $\left(C_{S}=3.70\right.$ and 3.16), and correlations $\left(R_{1}=0.32\right.$ and $0.38 ; R_{2}=0.14$ and 0.21$)$. In the

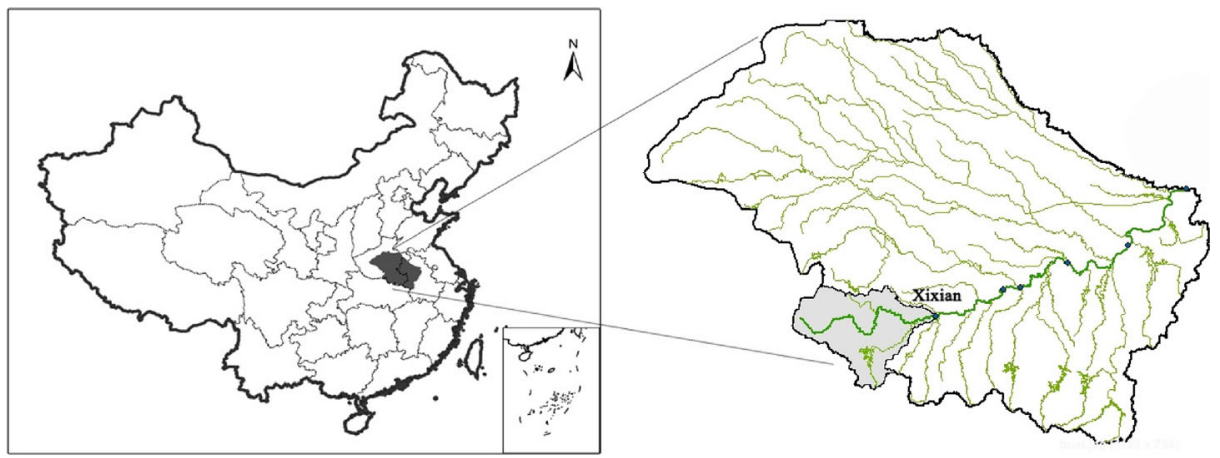

Fig. 1 Location of the Xixian hydrologic station in the Huai River watershed 
calibration period, runoff data fall within the range of $3.27-1560.00 \mathrm{~m}^{3} / \mathrm{s}$, and those in verification period fall within the range of $4.16-1030.00 \mathrm{~m}^{3} / \mathrm{s}$, being smaller than the former.

\subsection{Discrete Wavelet Decomposition Result}

The RS1 series is decomposed by the method of significance testing of DWT using "db8" mother wavelet and decomposition level $8\left(\log _{2} 492\right)$. Its energy function is compared with the reference energy function with $95 \%$ confidence interval, and the wavelet decomposition result is shown in Fig. 2. The sub-signals of RS1 series under "D" levels are reconstructed by detail wavelet coefficients, and the sub-signal under " $\mathrm{A}$ " level is reconstructed by approximation wavelet coefficients. The sub-signals of RS1 series under D1, D6, D7 and D8 have the energies falling within $95 \%$ confidence interval, so they are thought as noise, and others are dominant components for modeling RS1 series. The removed noise show pure random characters, and those identified deterministic components keep the dominant characters of original series, so we think that the "db8" wavelet used is suitable, and the wavelet decomposition results are reliable.

Both 1-month- and 3-month-ahead modeling of SR1 series are conducted. The proposed approach, conventional wavelet regression model and the AM-MCMC-WR (adaptive MetropolisMarkov chain Monte Carlo-wavelet regression) model proposed in (Sang et al. 2013) are used for comparison. In the AM-MCMC-WR model, the AM-MCMC algorithm is used to estimate parameters uncertainties in WR model, based on which probabilistic forecasting of hydrologic time series can be done. Three indices, RMSE (root mean square error), AARE (average absolute relative error) and $R^{2}$ (coefficient of determination), are used to evaluate the forecasting results:

$$
\begin{aligned}
& R M S E=\sqrt{\frac{1}{N} \sum_{i=1}^{N}\left[f^{\prime}(i)-f(i)\right]^{2}} \\
& A A R E=\frac{1}{N} \sum_{i=1}^{N}\left|\frac{f^{\prime}(i)-f(i)}{f(i)}\right| \times 100 \% \\
& R^{2}=\frac{\sum_{i=1}^{N}\left(f(i)-f^{\prime}(i)^{N}\right)^{2}}{\sum_{i=1}^{N}\left(f(i)-f^{\prime}(i)\right)^{2}+\sum_{i=1}^{N}\left(f^{\prime}(i)-f^{\prime}(i)\right)^{2}}
\end{aligned}
$$

Table 1 Statistical characters of monthly runoff data sets measured at the Xixian hydrologic station ${ }^{\mathrm{a}}$

\begin{tabular}{llllllll}
\hline Data set & \multicolumn{1}{l}{ Statistical characters } \\
\cline { 2 - 8 } & $\mathrm{x}_{\text {mean }}\left(\mathrm{m}^{3} / \mathrm{s}\right)$ & $\mathrm{x}_{\min }\left(\mathrm{m}^{3} / \mathrm{s}\right)$ & $\mathrm{x}_{\max }\left(\mathrm{m}^{3} / \mathrm{s}\right)$ & $\mathrm{C}_{\mathrm{v}}$ & $\mathrm{C}_{\mathrm{s}}$ & $\mathrm{R}_{1}$ & $\mathrm{R}_{2}$ \\
\hline Calibration (1961-1990) & 119.07 & 3.27 & 1560.00 & 1.43 & 3.70 & 0.32 & 0.14 \\
Verification (1991-2001) & 103.31 & 4.16 & 1030.00 & 1.54 & 3.16 & 0.38 & 0.21 \\
Whole (1961-2001) & 114.84 & 3.27 & 1560.00 & 1.46 & 3.59 & 0.34 & 0.16 \\
\hline
\end{tabular}

${ }^{\mathrm{a}} x_{\text {mean }}, x_{\text {min }}, x_{\max }, C_{v}, C_{s}, R_{1}$ and $R_{2}$ denote the mathematical mean, minimum, maximum, coefficient of variance, coefficient of skewness, lag-1 and lag-2 autocorrelation coefficients, respectively 

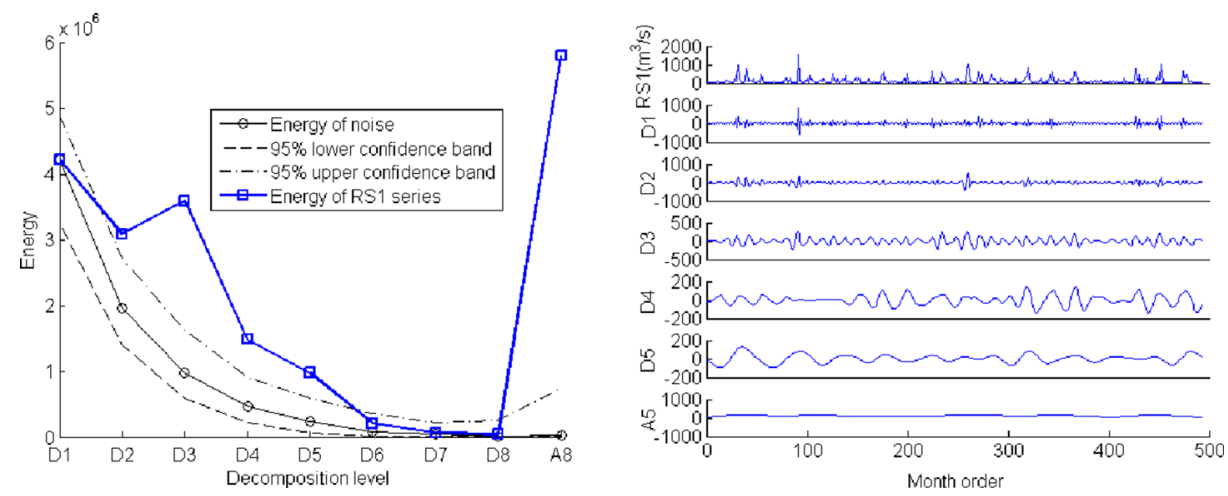

Fig. 2 Energy function (left) of the RS1 series and the reference energy function with $95 \%$ confidence interval, and discrete wavelet decomposition result of the RS1 series (right)

where $f(i)$ is observed data and $f^{\prime}(i)$ is modeled data with the total number of $N ; \overline{f^{\prime}(i)}$ is the mean of $f^{\prime}(i)$.

\subsection{Results and Discussion}

The input vectors of wavelet neural model are determined by analyzing partial correlations of RS1 series. Figure 3 shows that partial correlation character of RS1 series is statistically significant at lag-1, so 1 month-lag data are used as the input vector of BPNN and linear regression model. When employing the wavelet-based BPNN model, the input data are the sub-signals of RS1 series at 1 month-lag under D2, D3, D4, D5 and A8, and the output data is original series data. The model structure is determined as "5-8-1" following the "trail and error" procedure. The modeling processes of RS1 series by the wavelet regression model and AM-MCMC-WR model can be found in Sang et al. (2013).

The convergence criterion in wavelet neural modeling is assigned as 0.001 and 0.005 for comparison, and the likelihood function in Eq. (5) is used for training wavelet neural structure. Then, 1-month-ahead probabilistic forecasting of RS1 series at the verification period is conducted (Fig. 4). The probabilistic forecasting results by different models are depicted in Fig. 5, and further evaluated in Table 2. Following these results several conclusions are gained:

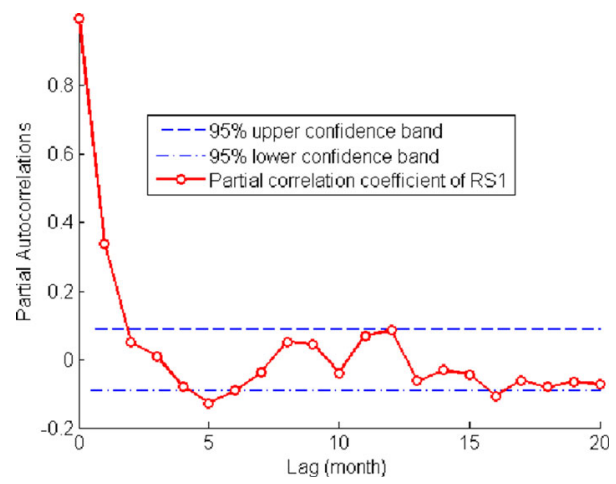

Fig. 3 Partial autocorrelation coefficients of the RS1 series with $95 \%$ confidence interval 
(1) The optimal results of 1-month-ahead forecasting by the proposed approach and the wavelet neural model are similar, and they are also similar with the observed hydrologic data, except some extreme values in the verification period (Fig. 5(a-b)). The results indicate that accurate forecasting result of RS1 series can be gained by the proposed approach and the wavelet neural model, but conventional neural model cannot do this, no matter which neural network structure is used. Because conventional neural model is to directly analyze raw hydrologic series, the results would be impacted by noise and insignificant components of original series. Comparatively, when firstly separating deterministic components from original series, the above influences can be effectively overcome, and the forecasting result of RS1 series can be improved by wavelet model.

(2) Figure 5(a-c) indicate that the wavelet neural model and wavelet regression model have similar performance in doing 1-month-ahead forecasting of RS1 series. Therefore, the proposed approach and the AM-MCMC-WR model, which are based on the former two respectively, also have similar performance for forecasting RS1 series. Scatterplots in Fig. 5(d) also show that three forecasting results are similarly close to the exact line.

(3) By using the proposed approach, $95 \%$ confidence interval of 1-month-ahead forecasting result of RS1 series in the verification period is obtained. Figure $5(\mathrm{a}-\mathrm{b})$ indicate that almost all original data (especially some extreme values) fall within the $95 \%$ confidence interval. However, conventional neural or wavelet neural model cannot do this. The $95 \%$ confidence interval estimated by the proposed approach and the $95 \%$ credible interval estimated by the AM-MCMC-WR model are similar. Since the latter is based on the Bayesian theory, which is widely used for uncertainty analysis, it is thought the proposed approach with the basic idea of resampling is effective.

(4) The resampling results of wavelet neural model for 1-month-ahead probabilistic forecasting of RS1 series (except Std. Dev. when $\mathrm{CC}=0.001$ ) become stable after 3000 times (Fig. 4). The result in Sang et al. (2013) indicates that the sampling number ensuring stable result should be bigger than 4000 by using the AM-MCMC-WR model. Comparatively, the proposed approach has higher efficiency in uncertainty evaluation than conventional Monte-Carlo method. It is due to the restraining effect of the convergence criterion. Because only those modeling results which meet the convergence criterion are accepted, the convergence rate of resampling processes is rapid.

(5) The one-month-ahead probabilistic forecasting results by using two convergence criterions ( 0.001 and 0.005$)$ are similar. Since optimal forecasting results of RS1 series can be gained using both the two convergence criterions, the two $95 \%$ confidence intervals estimated are also similar. It is concluded that uncertainty level of forecasting result is determined by the accuracy of forecasting result. If accurate forecasting result cannot be gained using a certain convergence criterion, it is not necessary to consider uncertainty again.

Three-month-ahead probabilistic forecasting results of RS1 series in the verification period are shown in Fig. 6, and the evaluation results are shown in Table 2. Compared with onemonth-ahead forecasting results, the 3-month-ahead forecasting results of RS1 series become worse. It is due to the less known knowledge of hydrologic processes for longer time-step forecasting.

The proposed approach and AM-MCMC-WR model also have similar performances for doing 3-month-ahead probabilistic forecasting of RS1 series. Figure 6 indicates that the $95 \%$ confidence interval estimated by the proposed approach can also cover almost all original series data, so uncertainty can be quantitatively estimated. However, $95 \%$ confidence interval 
(a)

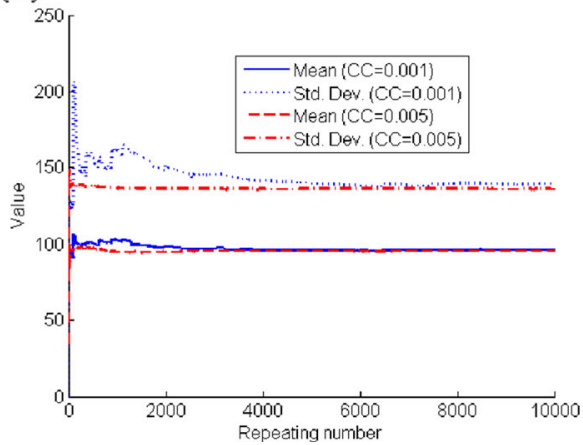

(b)

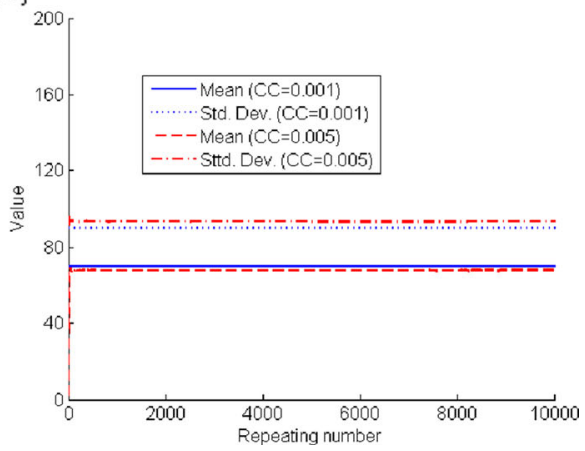

Fig. 4 Variation of training processes of the wavelet neural model for 1-month-ahead (a) and 3-month-ahead (b) probabilistic forecasting of the RS1 series. "CC" indicates convergence criterion, and "Std. Dev." indicates standard deviation

of the 3-month-ahead forecasting is bigger than that of 1-month-ahead forecasting, indicating more obvious uncertainty of 3-month-ahead forecasting. It is also due to the less known knowledge of hydrologic processes for longer time-step forecasting. Generally, along with the

(a)

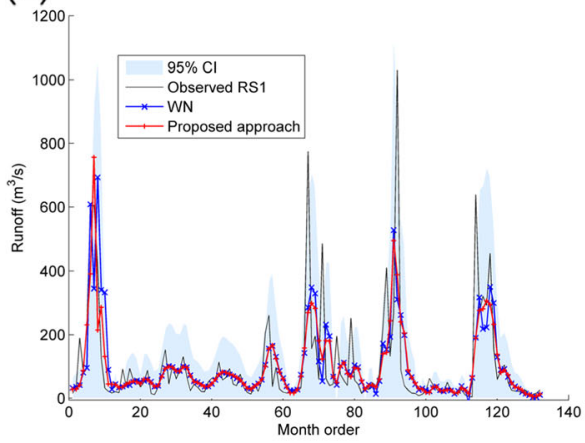

(c)

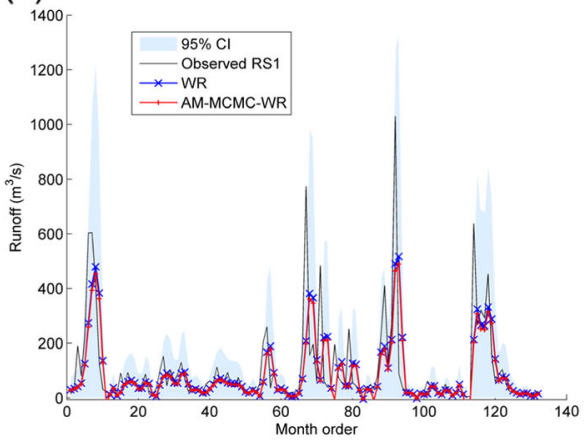

(b)

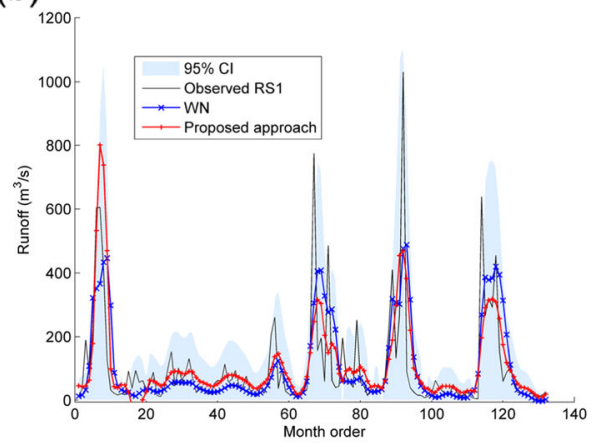

(d)

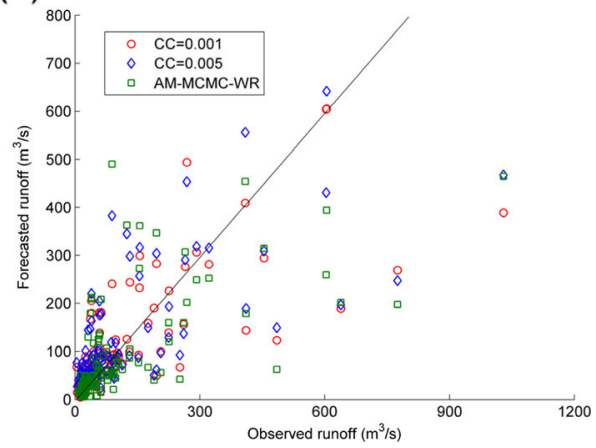

Fig. 5 Results of 1-month-ahead probabilistic forecasting of the RS1 series in the verification period using the convergence criterion $(\mathrm{CC}) 0.001$ (a) and 0.005 (b), probabilistic forecasting result by the AM-MCMC-WR model (c), and scatterplots of three forecasting results (d). In Figs. 5 and 6, "CI" indicates credible interval, "WN" indicates wavelet neural model, and "WR" indicates wavelet regression model 
Table 2 Comparison of the 1-month- and 3-month-ahead forecasting results of the RS1 series using the proposed approach and the AM-MCMC-WR model ${ }^{\mathrm{a}}$

\begin{tabular}{|c|c|c|c|c|c|c|c|}
\hline \multirow[t]{2}{*}{ Forecasting period } & \multirow[t]{2}{*}{ Model used } & \multicolumn{3}{|c|}{ Index (calibration) } & \multicolumn{3}{|c|}{ Index (verification) } \\
\hline & & $\operatorname{RMSE}\left(\mathrm{m}^{3} / \mathrm{s}\right)$ & AARE & $\mathrm{R}^{2}$ & $\operatorname{RMSE}\left(\mathrm{m}^{3} / \mathrm{s}\right)$ & AARE & $\mathrm{R}^{2}$ \\
\hline \multirow[t]{3}{*}{ 1-month-ahead } & $\mathrm{WN}(\mathrm{CC}=0.001)$ & 70.12 & 0.407 & 0.825 & 112.19 & 0.461 & 0.640 \\
\hline & $\mathrm{WN}(\mathrm{CC}=0.005)$ & 71.01 & 0.411 & 0.819 & 121.93 & 0.500 & 0.625 \\
\hline & AM-MCMC-WR & 70.28 & 0.413 & 0.823 & 119.48 & 0.466 & 0.658 \\
\hline \multirow[t]{3}{*}{ 3-month-ahead } & $\mathrm{WN}(\mathrm{CC}=0.001)$ & 84.36 & 0.478 & 0.749 & 133.36 & 0.503 & 0.614 \\
\hline & $\mathrm{WN}(\mathrm{CC}=0.005)$ & 86.12 & 0.501 & 0.731 & 151.93 & 0.524 & 0.552 \\
\hline & AM-MCMC-WR & 84.72 & 0.494 & 0.754 & 138.09 & 0.522 & 0.657 \\
\hline
\end{tabular}

a "CC" indicates convergence criterion; "WN" indicates wavelet neural model which is the basis of the approach proposed in this paper

increase of forecasting period, the relation among explanatory hydrologic data and forecasted hydrologic variable would become less known and weaker, so uncertainty would become more obvious, leading to worse forecasting results.

(a)

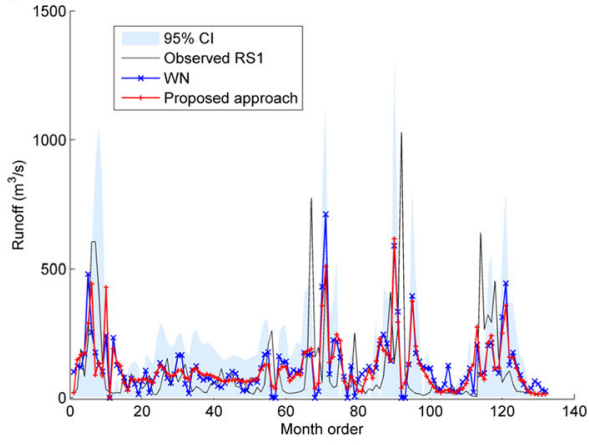

(c)

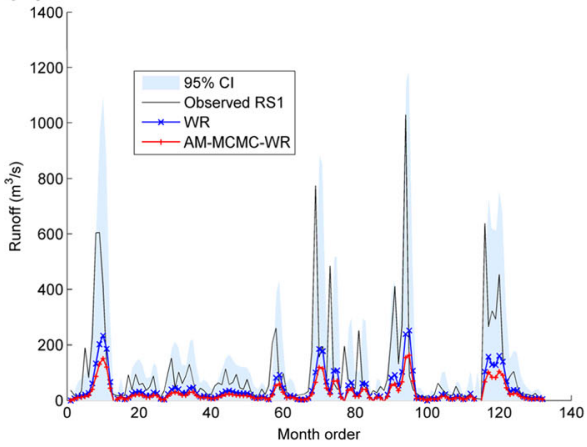

(b)

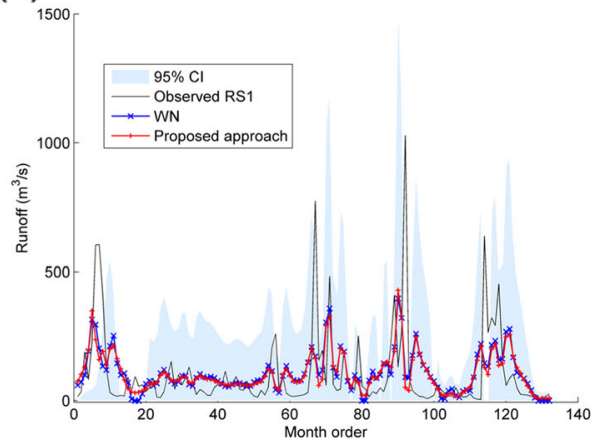

(d)

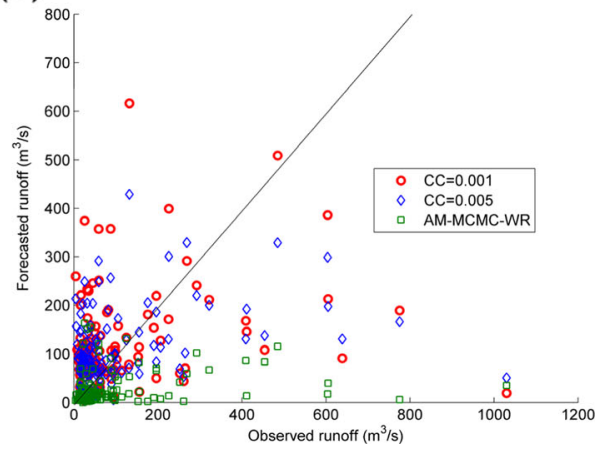

Fig. 6 Results of 3-month-ahead probabilistic forecasting of the RS1 series in the verification period using the convergence criterion (CC) 0.001 (a) and 0.005 (b), probabilistic forecasting result by the AM-MCMC-WR model (c), and scatterplots of three forecasting results $(\mathbf{d})$ 
In addition, the 3-month-ahead probabilistic forecasting by the proposed approach has identically rapid convergence rate as that in 1-month-ahead probabilistic forecasting, and it is also due to the effect of convergence criterion. However, the result in Sang et al. (2013) indicated slower convergence rate of 3-month-ahead probabilistic forecasting when using the AM-MCMC-WR model. Comparatively, the proposed approach has higher efficiency for probabilistic forecasting of hydrologic time series.

\section{Conclusions}

In this paper, an approach for probabilistic forecasting of hydrologic time series by wavelet neural model was proposed. By applying to the Xixian hydrologic station in the Huai River watershed for 1-month- and 3-month-ahead forecasting, the performance of the proposed approach was investigated. It can gain both optimal and probabilistic forecasting result by using wavelet neural model, so uncertainty can be evaluated, based on which more rational decisions can be made.

There are equifinality among neural parameters sets, so uncertainty is the inevitable existence in hydrologic time series forecasting by neural models. The understandings gained from this study are summed up as: (1) forecasting results become worse along with the increase of forecasting period. It is due to the less known knowledge about the relation between input and output data sets for longer-step forecasting; (2) choice of suitable convergence criterion is a key factor when using the proposed approach, because it directly determines the accuracy, convergence rate and uncertainty degree of probabilistic forecasting result. Generally, when the chosen convergence criterion can ensure the accuracy of optimal forecasting result, the proposed approach has high efficiency as Bayesian method, and both of them have much higher efficiency than conventional Monte-Carlo method. However, several detailed issues should be carefully considered when using the proposed approach, including the choice of proper likelihood function, accurate wavelet decomposition of series, and determination of proper wavelet neural structure. Only by carefully considering these issues, more reasonable results of hydrologic forecasting could be obtained.

Finally, it should be pointed out that although the proposed approach can do probabilistic forecasting, forecasting of extreme values is still a difficult task, which are clearly shown in the forecasting result of RS1 series, as well as many other present studies (Minns and Hall 1996; Jain and Kumar 2007). For the RS1 series, although the value range in the calibration data set can contain that in the verification data set, accurate forecasting result of extreme values cannot be obtained by any of those models used. Following stochastic hydrology theory (Chou 2011; Sang et al. 2014), deterministic components and extreme values in hydrologic series are generated by obviously different physical mechanisms (Kuczera 1992). Hydrologic extreme values usually show pure random characters and thus could not be accurately forecasted by any deterministic model. To improve hydrologic time series forecasting, it may be more feasible and desirable to describe the uncertainty of hydrologic extreme values by proper confidence interval.

Acknowledgments The authors gratefully acknowledged the helpful review comments and suggestions given by George P. Tsakiris, Editor-in-Chief, and two anonymous reviewers. The authors also thanked Ms. Feifei Liu for her assistance in the preparation of the manuscript. This project was financially supported by the Strategic Priority Research Program of the Chinese Academy of Sciences (No. XDB03030202), and the National Natural Science Foundation of China (No. 41330529, 41201036, 41271048). 


\section{References}

Alvisi S, Franchini M (2011) Fuzzy neural networks for water level and discharge forecasting with uncertainty. Environ Model Softw 26:523-537

Anctil F, Tape DG (2004) An exploration of artificial neural network rainfall-runoff forecasting combined with wavelet decomposition. J Environ Eng Sci 3:S121-S128

Arabi M, Govindaraju RS, Hantush MM (2007) A probabilistic approach for analysis of uncertainty in the evaluation of watershed management practices. J Hydrol 333(2-4):459-471

Beven K, Binley A (1992) The future of distributed model: model calibration and uncertainty prediction. Hydrol Process 6:279-298

Beven K, Freer J (2001) Equifinality, data assimilation, and uncertainty estimation in mechanistic modeling of complex environmental systems using the GLUE methodology. J Hydrol 249:11-29

Biemans H, Hutjes RWA, Kabat P, Strengers BJ, Gerten D, Rost S (2009) Effects of precipitation uncertainty on discharge calculations for main river basins. J Hydrometeorol 10(4):1011-1025

Bormann H (2008) Sensitivity of a soil-vegetation-atmosphere-transfer scheme to input data resolution and data classification. J Hydrol 351(1-2):154-169

Box GEP, Jenkins GM (1976) Time series analysis: forecasting and control. Holden-Day Publications, San Francisco

Cheng CT, Xie JX, Chau KW, Layeghifard M (2008) A new indirect multi-step-ahead forecasting model for a long-term hydrologic forecasting. J Hydrol 361:118-130

Chou CM (2011) A threshold based wavelet de-noising method for hydrological data modeling. Water Resour Manag 25(7):1809-1830

Coulibaly P, Anctil F, Bobee B (2000) Daily reservoir inflow forecasting using artificial neural networks with stopped training approach. J Hydrol 230(3-4):244-257

Danbechins I (1992) Ten lectures on wavelets. SIAM, Philadelphia

Geza M, Poeter EP, McCray JE (2009) Quantifying predictive uncertainty for a mountain-watershed model. J Hydrol 376(1-2):170-181

Jain A, Kumar AM (2007) Hybrid neural network models for hydrologic time series forecasting. Appl Soft Comput 7:585-592

Kaheil YH, Gill MK, McKee M, Bastidas L (2006) A new Bayesian recursive technique for parameter estimation. Water Resour Res 42(8):W08423

$\mathrm{Ki}$ i O (2009a) Neural network and wavelet conjunction model for modeling monthly level fluctuations of Van Lake in Turkey. Hydrol Process 23(14):2081-2092

$\mathrm{Ki}$ i O (2009b) Wavelet regression model as an alternative to neural networks for monthly streamflow forecasting. Hydrol Process 23:3583-3597

Ki i O (2010) Wavelet regression model for short-term streamflow forecasting. J Hydrol 389:344-353

Krzysztofowicz R (1999) Bayesian theory of probabilistic via deterministic hydrologic model. Water Resour Res 35(9):2739-2750

Krzysztofowicz R (2001) The case for probabilistic forecasting in hydrology. J Hydrol 249:2-9

Kuczera G (1992) Uncorrelated measurement error in flood frequency inference. Water Resour Res 28(1):183188

Kumar DN, Maity R (2008) Bayesian dynamic modeling for nonstationary hydroclimatic time series forecasting along with uncertainty quantification. Hydrol Process 22:3488-3499

Kwon HH, Lall U, Khalil AF (2006) Stochastic simulation model for nonstationary time series using an autoregressive wavelet decomposition: Applications to rainfall and temperature. Water Resour Res 42: W11404

Luk KC, Ball JE, Sharma A (2000) A study of optimal model lag and spatial inputs to artificial neural network for rainfall forecasting. J Hydrol 227(1):56-65

Maheswaran R, Khosa R (2014) A wavelet-based second order nonlinear model for forecasting monthly rainfall. Water Resour Manag 28(15):5411-5431

Maier HR, Dandy GC (2000) Neural networks for the prediction and forecasting of water resources variables: a review of modelling issues and applications. Environ Model Softw 15:101-124

Mallat S (1989) Multiresolution approximations and wavelet orthonommal bases of $\mathrm{L}^{2}(\mathrm{R})$. Trans Amr Math Soc 315(1):69-87

Minns AW, Hall MJ (1996) Artificial neural networks as rainfall-runoff models. Hydrol Sci J 41(3):399-417

Nourani V, Mogaddam AA, Nadiri AO (2008) An ANN-based model for spatiotemporal groundwater level forecasting. Hydrol Process 22:5054-5066

Nourani V, Alami MT, Aminfar MH (2009) A combined neural-wavelet model for forecasting of Ligvanchai watershed precipitation. Eng Appl Artif Intel 22:466-472

Percival DB, Walden AT (2000) Wavelet methods for time series analysis. Cambridge University Press, Cambridge 
Ramana RV, Krishna B, Kumar SR, Pandey NG (2013) Monthly rainfall prediction using wavelet neural network. Water Resour Manag 27(10):3697-3711

Sang YF (2012) A practical guide to discrete wavelet decomposition of hydrologic time series. Water Resour Manag 26(11):3345-3365

Sang YF (2013) A review on the applications of wavelet transform in hydrologic time series analysis. Atmosph Res 122:8-15

Sang YF, Wang D, Wu JC (2010) Probabilistic forecast and uncertainty assessment of hydrologic design values using Bayesian theories. Hum Ecol Risk Assess 16(5):1184-1207

Sang YF, Shang LY, Wang ZG, Liu CM, Yang MG (2013) Bayesian-combined wavelet regressive modeling for hydrologic time series forecasting. Chin Sci Bull 58:3796-3805

Sang YF, Liu CM, Wang ZG, Wen J, Shang LY (2014) Energy-based wavelet de-Noising of hydrologic time series. PLoS ONE 9(10): 110733

Shrestha DL, Solomatine DP (2006) Machine learning approaches for estimation of prediction interval for the model output. Neural Netw 19:225-235

Sivakumar B, Berndtsson R, Persson M (2001) Monthly runoff forecasting using phase space reconstruction. Hydrol Sci J 46(3):377-387

Tang Y, Reed P, van Werkhoven K, Wagener T (2007) Advancing the identification and evaluation of distributed rainfall-runoff models using global sensitivity analysis. Water Resour Res 43(6):W06415

Tiwari MK, Chatterjee C (2010) Uncertainty assessment and ensemble flood forecasting using bootstrap based artificial neural networks (BANNs). J Hydrol 382(1-4):20-33

Torrence C, Compo GP (1998) A practical guide to wavelet analysis. Bull Am Meteorol Soc 79(1):61-78

Tung WH, Mays LW (1981) Risk models for flood levee design. Water Resour Res 17(4):833-841

van Werkhoven K, Wagener T, Reed P, Tang Y (2009) Sensitivity-guided reduction of parametric dimensionality for multi-objective calibration of watershed models. Adv Water Resour 32(8):1154-1169

Vrugt JA, ter Braak CJF, Gupta HV, Robinson BA (2009) Equifinality of formal (DREAM) and informal (GLUE) Bayesian approaches in hydrologic modeling? Stoch Env Res Risk A 23(7):1011-1026

Wang W, Hu S, Li Y (2011) Wavelet transform method for synthetic generation of daily streamflow. Water Resour Manag 25(1):41-57 